\section{Simple-Box-9 coil system: \\ A novel approach to design of a square coil system for producing uniform magnetic fields}

Kunihisa Tashiro ${ }^{\mathrm{a}, \mathrm{b}}$, Akihito Matsuoka ${ }^{\mathrm{a}}$, and Hiroyuki Wakiwaka ${ }^{\mathrm{a}, \mathrm{b}}$

${ }^{a}$ Department of Electrical and Electronic Engineering, Shinshu University,

4-17-1 Wakasato, Nagano, 380-8553 Nagano, Japan

${ }^{\mathrm{b}}$ Spin Device Technology Center, Shinshu University, 4-17-1 Wakasato, Nagano, 380-8553 Nagano, Japan

Abstract: This paper presents a novel approach for the design of square coil system, through considerations of a conventional square coil system. This simple approach allows us to design a coil system which contains a greater number of coils. From this design method, we introduce a new structure system of same-sized square coils, Simple-Box-9. The constant coil spacing is a fourth-part of the side length of the coil, and the number of windings is $2 / 1 / 1 / 1 / 1 / 1 / 1 / 1 / 2$. From numerical calculations and experiments, it is shown that Simple-Box-9 can achieve the best uniformity compared with conventional square coil systems.

Keywords: uniform magnetic field, square coil system, design method

\section{Introduction}

Well-controlled, uniform magnetic field generation over a considerable volume is a necessary technique. A calibration instrument for a high sensitivity magnetometer, a geomagnetic field canceling system for biomagnetic measurements, a human motion capturing system with magnetometers, and a nuclear magnetic resonance imaging system at ultralow field are good examples. Although the required amplitude is not so high $(\sim 100 \mu \mathrm{T})$, the volume should be capable of a human. And the required uniformity is at least within $1 \%$ deviation. This paper focuses on same-sized square coil systems which have practical advantages in construction and usefulness. Most of the previous works proposed configurations which would zero as many high-order spatial derivatives of the magnetic field as possible at the center of the coil system[1-2]. Another method discussed the uniformity in an evaluation area inside the equally-spaced coils [3-5]. First of all, we categorize the conventional design method into $\mathrm{A}$ and $\mathrm{B}$, and discuss the difficulty of the optimization. In the next step, we propose a novel approach for the design of a square coil system which involves the advantages of Category A and B. Using this method, the number of parameters which should be defined is only two. From this approach, we lead to a new square coil system structure, Simple-Box-9, and evaluate its capability through numerical calculations and experiments.

\section{Conventional square coil system}

2.1 Category A

Consider a square coil system which is coaxially placed $N$ square coils of side length $d$ [m], as shown in Fig. 1. Here, the $x$ and $y$ axes are parallel to the side of coils, the $z$ axis is the center axis of the coil system, and the center of the system represents $(x, y$, $z)=(0,0,0)$. A square loop carrying a current $I[\mathrm{~A}]$, which is placed at a distance $c[\mathrm{~m}]$ from the center, produces a magnetic flux density $B(z, c)$ at $(0,0, z)$. From Biot-sarvart's law, $B(z, c)$ has a $z$ component only and can be expressed as follows

$$
B(z, c)=\frac{2 \times 10^{-7} \times I d^{2}}{\left(\frac{d^{2}}{4}+(z-c)^{2}\right) \sqrt{\frac{d^{2}}{2}+(z-c)^{2}}}[\mathrm{~T}]
$$

When $N$ square loops carrying $I_{\mathrm{i}}$ at a distance $c_{\mathrm{i}}$ are considered, the total magnetic flux density $B(z)$ can be calculated by

$$
B(z)=\sum_{i=1}^{N} B\left(z, c_{i}\right) \quad[\mathrm{T}]
$$

Considering the Taylor-series expansion for $B(\mathrm{z})$ with respect to the center,

$$
B(z)=\sum_{i=0}^{\infty} a_{i} z^{i} \quad[\mathrm{~T}]
$$

Assuming symmetry of the coil arrangement with the $z=0$ plane, and giving the same current to a pair of square loops,

$$
B(z)=\sum_{i=0}^{\infty} a_{(2 i)} z^{(2 i)}
$$

$B(\mathrm{z})$ is an even function of $z$, and all odd-order terms vanish. Since the parameters which should be determined are $d, c_{\mathrm{i}}$ and $I_{\mathrm{i}}$, the number of parameters is $(2 N-1)$. When each square coil has a number of windings $n_{\mathrm{i}}$, and all of coils are connected in series, $n_{\mathrm{i}}$ is an alternative parameter of $I_{\mathrm{i}}$. To be the minimum number of parameters, ampere-turn ratio $\left(I_{\mathrm{i}} / I_{\mathrm{j}}\right)$, and normalized distance $(z / d)$ are introduced. Here, $I_{\mathrm{j}}$ refers to the ampere-turn of the innermost coil. When the number of $N$ is odd, the number of coil pairs is $((N-1) / 2)$, and an innermost coil is placed at the center. The number of parameters for the normalized distance is $((N-1) / 2)$, and for the ampere-turn ratio it is $((N-1) / 2)$. When the number of $N$ is even, the number of coil pairs is $(N / 2)$. The number of parameters for the normalized distance is $(N / 2)$, and for the ampere-turn ratio it is $((N / 2)-1)$. It means that all cases require the total number of parameters is to be $(N-1)$. The parameters can be 
defined by the solution of

$$
\left.\frac{d^{(2 i)} B(z)}{d z^{(2 i)}}\right|_{z=0}=0 \quad(\text { Where } i=1, \ldots,(N-1))
$$

According to the design method (Category A), every term up to the $(2 N-1)$-order will vanish. Equation (5) can be solved analytically, and a solution for up to $N=4$ was calculated. Proposed structure based on Category A is summarized in Table 1. Because the normalized distance and the ampere-turn ratio were irrational number for $N=3$ and 4 [1], the normalized distance with four digits, and the integer number of turns of coil system were proposed [2].

\subsection{Category B}

For reducing the number of parameters, equally-spaced square coil systems are considered. To give the same coil spacing $s[\mathrm{~m}]$, the parameter which should be determined is the ampere-turn ratio. Using this design method (Category B), the uniformity of the axial field in an evaluation area is a condition for finding a suitable ampere-turn ratio. A proposed structure based on Category B is summarized in Table 2. Simple-Box-9 is explained in the next chapter.

In the case of $N=3$, Grisenti's group defined a few axes parallel to the center axis of the evaluation area [3]. For $s=(2 d / 5)$, they proposed an ampere-turn ratio of 0.6. This structure is a simplified structure of Merrit-3[2]. Moreover, they noted that the cube-surface coil is suitable for a three-axis coil system, and a proposed ampere-turn ratio of 0.51 for $s=(d / 2)$. From the view point of practical use, the authors proposed the number of windings as $2 / 1 / 2$ [4]. In the case of same coil arrangement, Sasada also proposed an ampere-turn ratio of 0.64 and the number of windings as 25/16/25 [5]. In this structure, the uniformity area within $2 \%$ deviation was $0.42 d \times 0.70 d$ in the $x-z$ plane.

In the case of $N=5$, Rubens chose $\mathrm{s}=(d / 4)$ to be the cube-surface coil [6]. In order to find the ampere-turn ratio of $a_{2}: a_{1}: 1: a_{1}: a_{2}$, giving the condition

$$
\begin{aligned}
B(z)= & B(z, 0)+a_{1}\left(B\left(z,-\frac{d}{4}\right)+B\left(z, \frac{d}{4}\right)\right) \\
& +a_{2}\left(B\left(z,-\frac{d}{2}\right)+B\left(z, \frac{d}{2}\right)\right)
\end{aligned}
$$

To solve this equation, $z=0,0.15 d$, and $0.25 d$ was chosen using a heuristic approach. It led to $a_{1}=0.405$ and $a_{2}=1.92$, and corresponded to the number of windings as $71 / 15 / 37 / 15 / 71$. In the next heuristic approach, it was found that the condition of $a_{1}=$ 0.400 and $a_{2}=1.900$ gives greater uniformity along the axis, thus $19 / 4 / 10 / 4 / 19$ as the number of windings was proposed.
2.3 Comparison of conventional square coil systems

For the evaluation of conventional square coil systems, this paper focuses on the uniformity of magnetic flux density along the center axis. The magnetic flux density $B(z)$ can be calculated from formula (1) and (2), and the deviation $e$ is defined by

$$
e=\frac{B(z / d)-B(0)}{B(0)} \times 100 \quad[\%]
$$

Fig. 2 shows the comparison of the uniformity between Category A and B. The vertical axis is a logarithmic scale, and represents absolute the value of the deviation $|e|$. The horizontal axis represents normalized distance $(z / d)$. In Category A, all curves were an increasing function of $(z / d)$, and showed a similar profile. Use of a greater number of coils made better uniformity area wide. Although all curves showed a different profile in Category B, we can see that the use of the number of coils could produce a more uniform area. Comparison of the uniformity on the $x-z$ plane for several coil systems which correspond to Helmholtz-2, Merritt-3, Merritt-4 and Rubens 5 had been done by a previous researcher [7]. This result agrees with the Kirshvink's proposal that Merrit-4 is the best. In order to improve the uniformity, coaxially arranged two sets of Merritt-4 was also proposed.

Since parameters can be found uniquely in the design based on Category A, the coil distance and the ampere-turn ratio will be an irrational number. Because the error of the approximation should be considered, it is not easy to optimize for practical structure when the number of coils is increased. In the design based on Category B, numerous answers will appear, which depend on the definition of the evaluating area and criteria.

From another point of view, the number of turns makes parasitic capacitance large, and the useful frequency range becomes small. Although an independent current source for driving each single winding coil could produce the desired magnetic field, the precise control of the current source is not easy. Simple-Cubic-3 which has simplest integer number of windings achieves the deviation of $2.6 \%$ within $d / 2$ area, and the area uniformity is better than that of Helmholtz-2. It was reported that Simple-Cubic-3 achieves uniformity within $2.6 \%$ deviation inside a sphere volume with $d / 2$ diameter [4]. This is a better deviation compared with other relatively simple coil systems, such as $3.7 \%$ of Cubic-3 and $7.7 \%$ of Helmholtz-2. It should be noted that a tripled Simple-Cubic-3 coil system is suitable for use in uniform magnetic field generation in three-dimensions. However, other design methods capable of using the number of coils should be considered when the object is not spherical or more uniformity is required. 


\section{Novel approach to design of a square coil system}

3.1 Design method

This paper proposes a novel approach to the design of a square coil system which involved the advantages of Category A and B. First of all, this approach limits the number of parameters such as Simple-Cubic-3 in Category B. This design accepts a coil spacing constant $s$, and sets the number of turns to be 2 and 1 for inner coils and two for outer coils, respectively. The two outer coils can be thought of as being used for end correction which is known in the design of a solenoid coil with finite length [8]. In this design, the parameters which should be defined are only $s$ and $N$. Next, comparison of the uniformity on the center axis helps to find the optimum parameters as in Category A. In order to find a candidate value of $s$ and the range of $N$, this design evaluates the center field $B(0)$, to which the coil system of infinite length and of finite length is balanced. Then, suitable $N$ is heuristically chosen through the calculation of the uniformity for given values of $s$.

\subsection{Center field $B(0)$}

Fig. 3 shows the center field $B(0)$ as a function of the number of coils $N$ and as a parameter of $s$. The initial value of $N$ is three, and the increment is two. An increase of $N$ causes $B(0)$ to increase in the case of $s=(d / 2)$, and causes $B(0)$ to decrease when $s$ is smaller than $(d / 4)$. The final value of $B(0)$ approaches $(2.5 / d),(5.0 / d)$ and $(10 / d)$ for $\mathrm{s}=(d / 2),(d / 4)$ and $(d / 8)$, respectively. We can see that the final value as inversely proportional to the coil distance is valid, because the magnetic flux density of an ideal solenoid coil is proportional to the ampere-turn per length. The smallest variation of the initial value to final value is about $10 \%$ at $s=(d / 4)$. This paper guesses that a suitable value of $s$ is $(d / 4)$ and the range of $N$ is less than 20 .

\subsection{Uniformity on the center axis}

Fig. 4 shows the deviation on the center axis as a function of the normalized distance and as parameters of the number of coils. The increment of $(z / d)$ is 0.1 , and the value of $s$ are $(d / 2)$ and $(d / 4)$. The initial value of $N$ is three, the increment is two, and the final value is nineteen. According to the range of deviation, uniform area is compared with the length of the coil system.

If the coil spacing is $(d / 2)$, uniform area within $\pm 3 \%$ deviation is $60 \%$ at $N=3$, and is $30 \%$ at $N=5$. For $N>7$, it becomes an increasing function which could be expressed as $(100 \times(2.8+(\mathrm{N}-7)) /(\mathrm{N}-1))[\%]$. $N=17$ and 33 correspond to the uniform area of $80 \%$ and $90 \%$, respectively. If $N>7$, use of a greater number of coils extends the uniform area within an oscillating $1 \%$ deviation, which could be expressed by $(100 \times(1.6+(\mathrm{N}-7) /(\mathrm{N}-1))[\%]$. In this case, $N=23$ and 45 corresponds to a uniform area of $80 \%$ and $90 \%$, respectively. Unfortunately, it was found that use of a greater number of coils does not expand the uniform area within $0.1 \%$ deviation. From the results, we can see that the end coils do not affect the uniformity, and the magnetic flux distribution is similar to that of a sparsely wound solenoid coil. Therefore, a better uniform volume inside the coil system is not promising.

If the coil spacing is $(d / 4)$, although uniform area within $\pm 3 \%$ deviation is only $20 \%$ at $N=3$, it becomes $80 \%$ at $N=9$. Although it is also an increasing function for $N>9$, a greater number of coils does not effectively expand the uniform area, for example, it is only $85 \%$ at $N=19$. At $N=9,11$, and 13 , uniformity area within $\pm 1 \%$ deviation is greater than $70 \%$, and a greater number of coils makes the area shrink. Furthermore, uniform area within $\pm 0.1 \%$ is $60 \%$ at $N=9$, and a uniform area which is greater than $30 \%$ is not found until $N=23$. From the results, this paper focuses on the coil system where $s=(d / 4)$ and $N=9$, and calls it Simple-Box-9.

\section{Evaluation of Simple-Box-9}

\subsection{Comparison of uniformity}

In order to evaluate the capability of Simple-Box-9, the uniformity on the center axis is compared with a conventional square coil system. Due to the best uniformity in Category A and B, Merritt-4 and Rubens-5 are chosen as the reference. Fig. 5 shows the comparison of uniform area within $\pm 1 \%$ deviation on the center axis as a function of normalized length $(2 z / l)$. In both Merritt-4 and Rubens-5, uniform area within $\pm 1 \%$ deviation is about $70 \%$ compared with the length of coil system. In contrast, it corresponds to $75 \%$ for Simple-Box-9. Since this coil system has twice the length of a coil system, the uniform area is fourfold compared with the conventional coil systems. According to the basis of Category A, a better uniformity on the center axis produces a better uniformity in the volume of the coil system. From these considerations, a better uniformity inside volume of Simple-Box-9 is expected.

\subsection{Uniformity on the $x-y$ plane}

Uniformity inside volume of Simple-Box-9 is calculated with a three-dimensional finite element method (3D FEM) program, COMSOL Multiphysics 3.5. Fig. 6 shows a result of the axial field in the $x-y$ planes at which the nine square coils are placed. The gray area indicates $\pm 1 \%$ deviation area compared with the center field. In this calculation, the number of elements was 407,694, the number of freedoms was 2,626,276. And the conditions are the limit for our PC with 4 GB memory. Although it looks rough calculation result, we can see that the Simple-Box-9 can achieve the uniform field in relatively large volume.

\author{
4.3 Uniformity on the $x-z$ plane \\ Uniformity inside volume of Simple-Box-9 is
}


confirmed by numerical analysis and experimentation. Fig. 7 shows the photograph of the developed Simple-Box-9 coil system. The side length of coil $d$ is $0.5 \mathrm{~m}$, and the number of windings is $2 / 1 / 1 / 1 / 1 / 1 / 1 / 1 / 2$. From formula (2), the center field is $4.762 / d[\mu \mathrm{T} / \mathrm{A}]$, which in this case is $9.524 \mu \mathrm{T} / \mathrm{A}$. A center field of $1 \mu \mathrm{T}$ at $100 \mathrm{~Hz}$ was generated, measured at a spacing of $0.1 d$ in the $x-z$ plane with a fluxgate sensor (uMAG-01N, MEDA), and then the absolute value of the deviation was evaluated. Because FEM results did not have enough accuracy, we directly calculated the field from Biot-sarvart's law with a software, Maple 10. The calculated and measured results are shown in Fig 8. The gray area represents the area within $1 \%$ deviation. From the numerical calculations, the uniform plane within $0.1 \%$ deviation is $0.4 d \times 0.9 d$, and that within $1 \%$ deviation is $0.6 d \times 1.4 d$ in the $x-z$ plane. It is the best uniformity compared with conventional square coil systems. The measured results are in agreement with it.

\section{Conclution}

(1) Conventional square coil systems were categorized into two, and the uniformity on the center axis was discussed for the evaluation.

(2) In both categories, use of the number of coils $N$ can achieve a better uniformity. However, an increase of the number of parameters makes optimization difficult.

(3) A novel approach to the design of a square coil system was presented, which involved the advantages of Category A and B.

(4) A new structure of a square coil system, Simple-Box-9, was led from the design.

(5) From numerical analysis and experimentation, Simple-Box-9 has the best uniformity compared with conventional square coil systems.

\section{References}

[1] K. Kakuno and Y. Gondo, Bulletin of the Faculty of Engineering, Yokohama National Univ., 25 179, (1976).

[2] R. Merritt, C. Purcell, and G. Stroink, Rev. Sci. Instrum. 54, 879 (1983).

[3] R. Grisenti and A. Zecca, Rev. Sci. Instrim. 52, 1097 (1981).

[4] K. Tashiro, and H. Wakiwaka, The Bioelectromagnetic Society 29th Annual Meating (BEMS2007), 421 (2007).

[5] I. Sasada, Magn. Soc. Japan 27, 612 (2003). in Japanese

[6] S. Rubens, Rev. Sci. Instrum., 16, 243 (1945).

[7] J. L. Kirschvink, Bioelectromagnetics 13, 401 (1992).

[8] B. Lewis, British Journal of Applied Physics 1, 238 (1950).

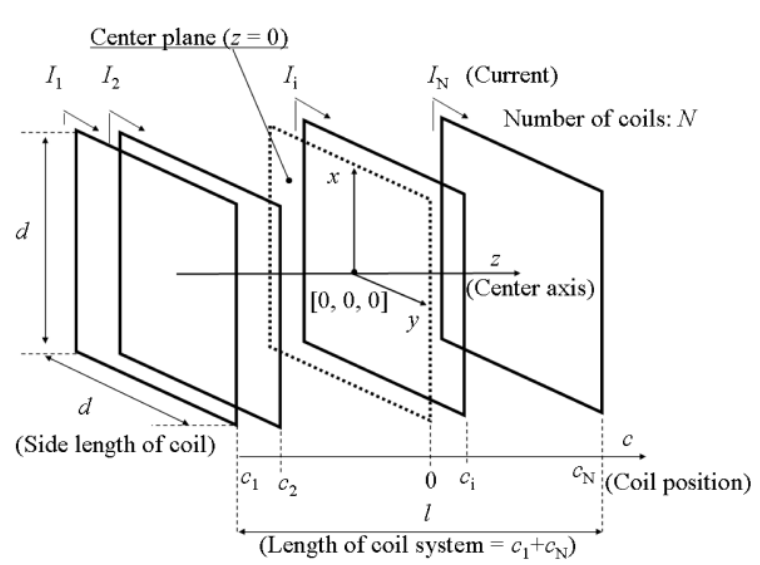

Fig. 1 Schematic arrangement of a square coil system.

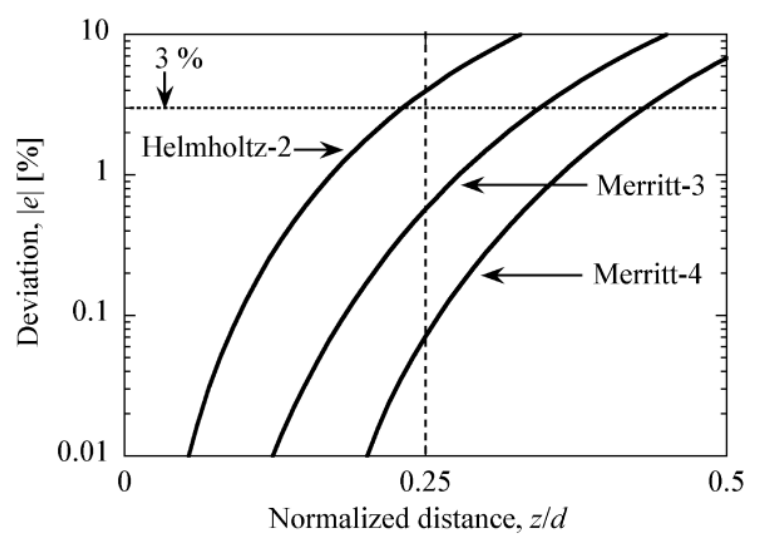

(a) Category $\mathrm{A}$

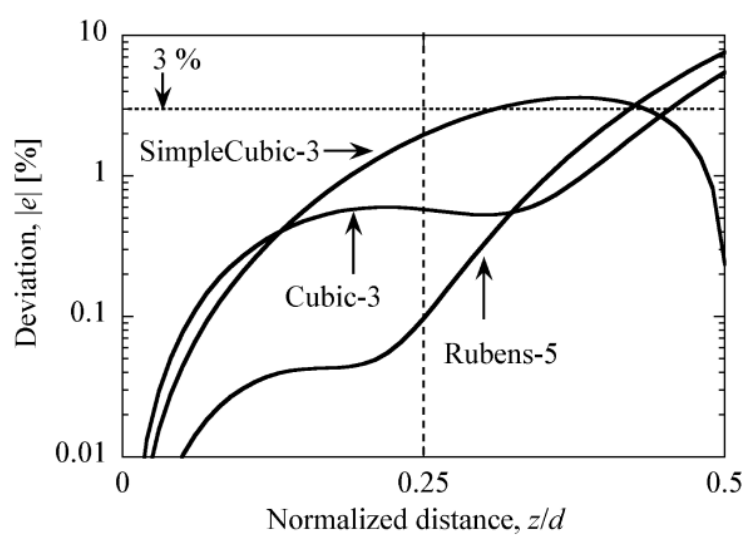

(b) Category B

Fig. 2 Comparison of the uniformity along the center axis between Category A and B. The parameter refers to the name of the coil system described in Table 1 and 2. 


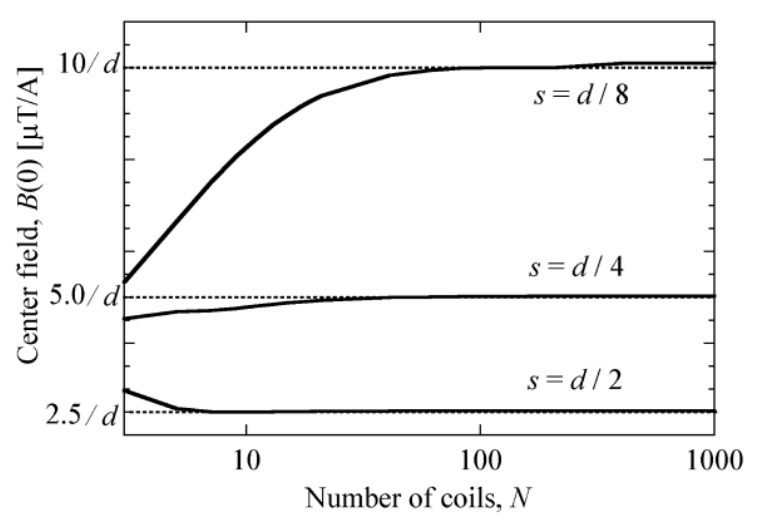

Fig. 3 Center field strength as a function of the number of coils and as a parameter of coil spacing.

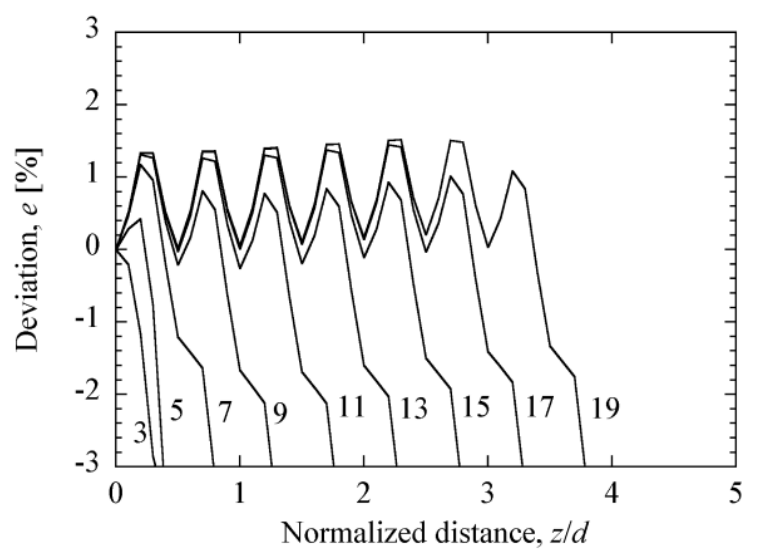

(a) $s=(d / 2)$

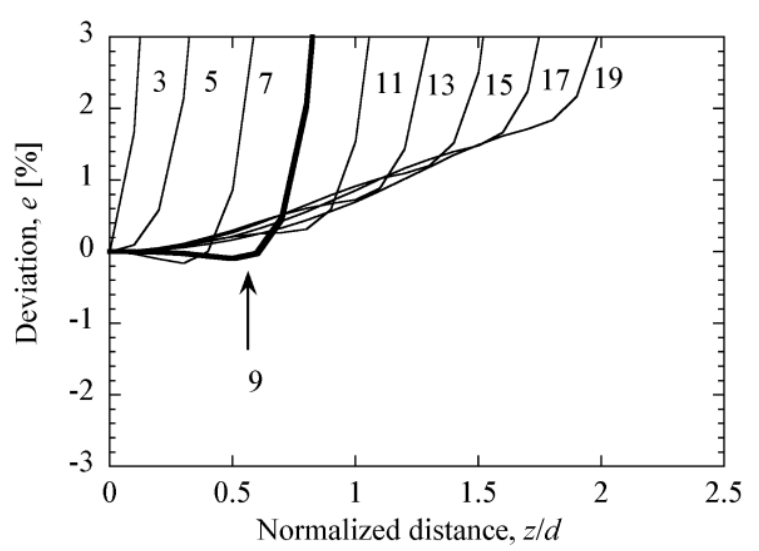

(b) $s=(d / 4)$

Fig. 4 Uniformity along the center axis as a function of normalized distance and as a parameter of the number of coils.

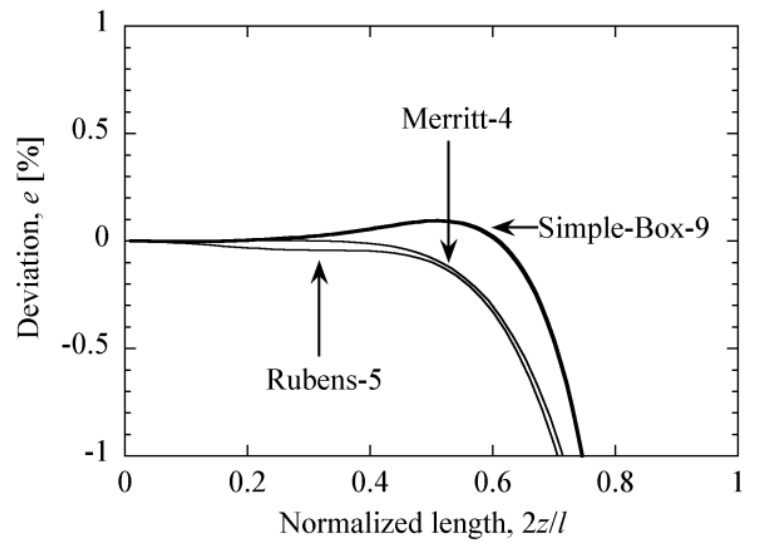

Fig. 5 Comparison of uniformity along the center axis as a function of normalized length. The parameter refers to the name of the coil system described in Table 1 and 2.

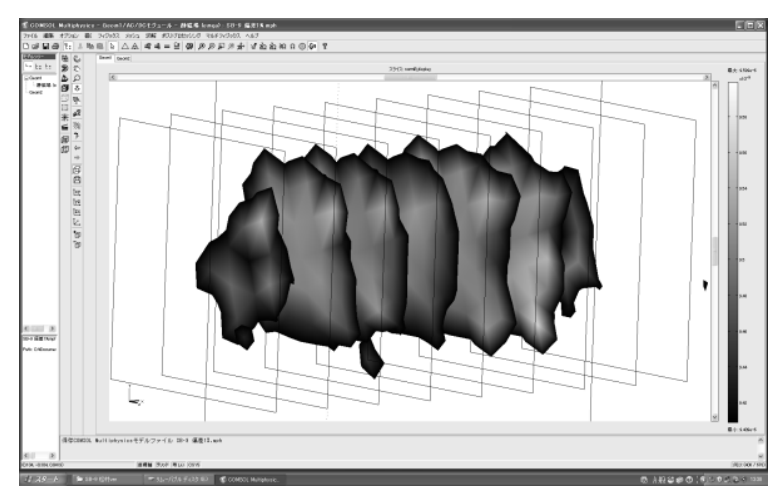

Fig. 6 An FEM analysis result of the axial field on the $x-y$ planes of Simple-Box-9. Nine $x-y$ planes are displayed, at which the square coils are placed. The gray area indicates $\pm 1 \%$ deviation area compared with the center field.

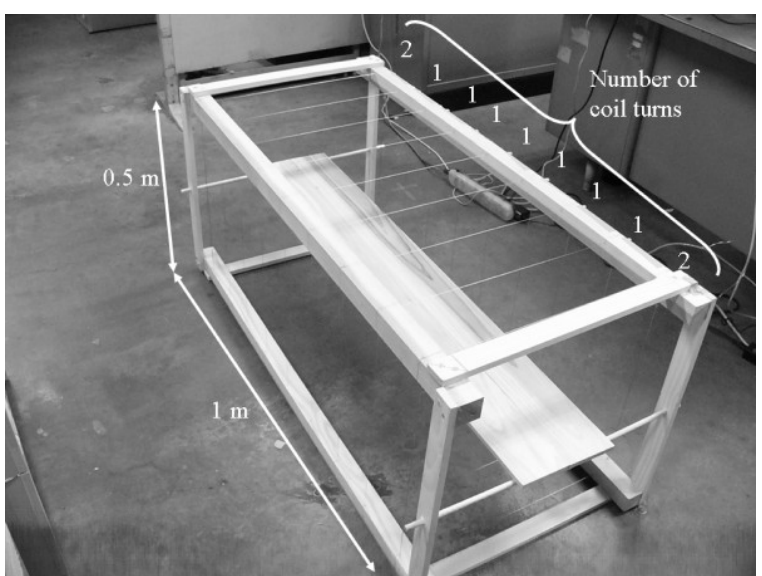

Fig. 7 Photograph of a developed Simple-Box-9 coil system. 


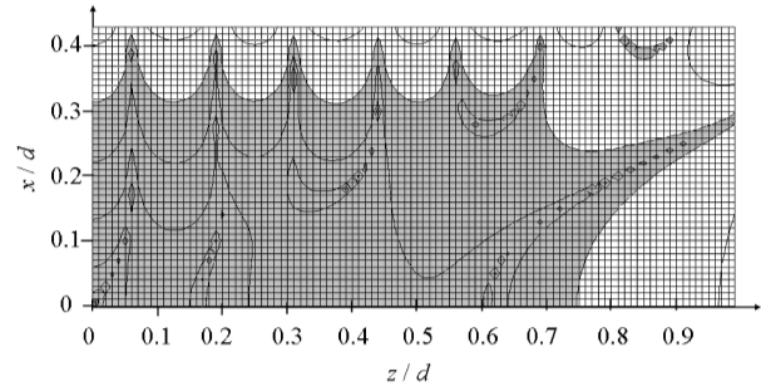

(a) Numerical calculations

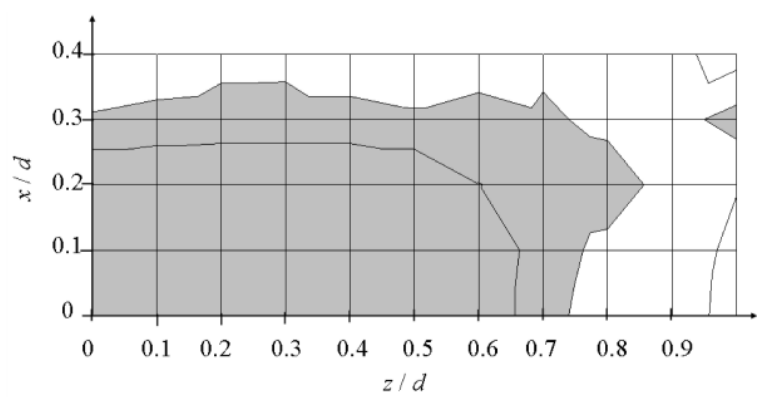

(b) Experimental results

Fig. 8. Uniformity of the axial field in the $x-z$ plane of Simple-Box-9. The gray area indicates $\pm 1 \%$ deviation area compared with the center field.

Table 1. Design of square coil systems in Category A [1-2]

\begin{tabular}{|c|c|c|c|c|c|c|}
\hline $\begin{array}{l}\text { Number } \\
\text { of coils, } \\
\qquad N\end{array}$ & $\begin{array}{l}\text { Length } \\
\text { of coil } \\
\text { system, } l\end{array}$ & $\begin{array}{l}\text { Coil distance with } \\
\text { respect to center of } \\
\text { system, } c_{i}\end{array}$ & $\begin{array}{l}\text { Ampere-turn ratio } \\
\text { of inner to } \\
\text { outer coil pair }\end{array}$ & $\begin{array}{c}\text { Proposed } \\
\text { number } \\
\text { of windings }\end{array}$ & $\begin{array}{l}\text { Center field, } \\
B(0)[\mu \mathrm{T} / \mathrm{A}]\end{array}$ & $\begin{array}{l}\text { Name of coil } \\
\text { system in this } \\
\text { paper }\end{array}$ \\
\hline 2 & $0.5445 d$ & $-0.27225 d,+0.27225 d$ & (N/A) & $1 / 1$ & $1.629 / d$ & Helmholtz-2 [1] \\
\hline \multirow{2}{*}{3} & \multirow{2}{*}{$0.8212 d$} & \multirow{2}{*}{$-0.4106 d, 0,+0.4106 d$} & \multirow{2}{*}{$0.512797 \ldots$} & $39 / 20 / 39$ & $68.21 / d$ & Merritt-3 [2] \\
\hline & & & & $33 / 17 / 33$ & $57.80 / d$ & $(\mathrm{~N} / \mathrm{A})[2]$ \\
\hline \multirow{3}{*}{4} & \multirow{3}{*}{$1.0110 d$} & \multirow{3}{*}{$\begin{array}{l}-0.5055 d,-0.1281 d \\
+0.5055 d,+0.1281 d\end{array}$} & \multirow{3}{*}{$0.423514 \ldots$} & $26 / 11 / 11 / 26$ & $46.65 / d$ & Merritt-4 [2] \\
\hline & & & & $59 / 25 / 25 / 59$ & $105.9 / d$ & $(\mathrm{~N} / \mathrm{A})[2]$ \\
\hline & & & & $85 / 36 / 36 / 85$ & $152.6 / d$ & $(\mathrm{~N} / \mathrm{A})[2]$ \\
\hline
\end{tabular}

$d[\mathrm{~m}]$ represents side length of coil.

Table 2. Design of square coil systems in Category B [3-5]

\begin{tabular}{|c|c|c|c|c|c|c|}
\hline $\begin{array}{l}\text { Number } \\
\text { of coils, } \\
N\end{array}$ & 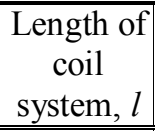 & $\begin{array}{c}\text { Coil spacing, } s \\
\quad\left(s=c_{\mathrm{j}+1}-c_{\mathrm{j}, .}\right. \\
\text { where } j=1, \ldots N-1)\end{array}$ & $\begin{array}{c}\text { Ampere-turn ratios } \\
\text { of innermost to } \\
\text { outer coil pair } \\
\end{array}$ & $\begin{array}{c}\text { Proposed number } \\
\text { of windings }\end{array}$ & $\begin{array}{l}\text { Center field, } \\
B(0)[\mu \mathrm{T} / \mathrm{A}]\end{array}$ & $\begin{array}{l}\text { Name of coil } \\
\text { system } \\
\text { in this paper } \\
\end{array}$ \\
\hline \multirow{4}{*}{3} & $0.8 d$ & $4 d / 5$ & 0.6 & (1/0.6/1) & $(1.880 / d)$ & $(\mathrm{N} / \mathrm{A})[3]$ \\
\hline & \multirow{3}{*}{$d$} & \multirow{3}{*}{$d / 2$} & 0.51 & $(1 / 0.51 / 1)$ & $(1.500 / d)$ & $(\mathrm{N} / \mathrm{A})$ [3] \\
\hline & & & 0.64 & $25 / 16 / 25$ & $41.20 / d$ & Cubic-3[5] \\
\hline & & & 0.5 & $2 / 1 / 2$ & $2.978 / d$ & SimpleCubic-3[4] \\
\hline \multirow{2}{*}{5} & \multirow{2}{*}{$d$} & \multirow{2}{*}{$d / 4$} & $0.405,1.92$ & $71 / 15 / 37 / 15 / 71$ & $133.0 / d$ & $(\mathrm{~N} / \mathrm{A})[5]$ \\
\hline & & & $0.400,1.900$ & $19 / 4 / 10 / 4 / 19$ & $35.69 / d$ & Rubbens-5[5] \\
\hline 9 & $2 d$ & $d / 4$ & $1,1,1,2$ & $2 / 1 / 1 / 1 / 1 / 1 / 1 / 1 / 2$ & $4.762 / d$ & SimpleBox-9 \\
\hline
\end{tabular}

$d[\mathrm{~m}]$ represents side length of coil. 